\title{
Selection of the Number of Components Using a Genetic Algorithm for Mixture Model Classifiers
}

\author{
Hiroshi Tenmoto ${ }^{1}$, Mineichi Kudo ${ }^{2}$, and Masaru Shimbo ${ }^{2}$ \\ 1 Department of Information Engineering, Kushiro National College of Technology, \\ Otanoshike Nishi 2-32-1, Kushiro, Hokkaido 084-0916, Japan \\ Tel: +81-154-57-7351 Fax: +81-154-57-6251 \\ tenmo@kushiro-ct.ac.jp \\ 2 Division of Systems and Information Engineering, Graduate School of Engineering, \\ Hokkaido University, Kita 13 Jo Nishi 8 Chome, Kitaku, Sapporo 060-8628, Japan \\ Tel/Fax: +81-11-706-6852 \\ \{mine, shimbo\}@main.eng.hokudai.ac.jp
}

\begin{abstract}
A genetic algorithm is employed in order to select the appropriate number of components for mixture model classifiers. In this classifier, each class-conditional probability density function can be approximated well using the mixture model of Gaussian distributions. Therefore, the classification performance of this classifier depends on the number of components by nature. In this method, the appropriate number of components is selected on the basis of class separability, while a conventional method is based on likelihood. The combination of mixture models is evaluated by a classification oriented MDL (minimum description length) criterion, and its optimization is carried out using a genetic algorithm. The effectiveness of this method is shown through the experimental results on some artificial and real datasets.
\end{abstract}

Keywords: mixture model classifier, class-conditional probability density function, class separability, minimum description length criterion, genetic algorithm

\section{Introduction}

In pattern recognition, we often apply the mixture model of Gaussian distributions to the approximation of a class-conditional probability density function. The mixture model is more expressive than simple distributions, so the Bayesian classifier based on the mixture models has higher classification performance than simple classifiers. In addition, this semiparametric classifier can absorb the statistical fluctuation of training samples, unlike nonparametric classifiers.

In the mixture model classifiers, the number of components directly affects the classification performance by nature. Nevertheless, in many applications, the number of components seems to be selected without careful consideration. One practical and reasonable selection method is based on MDL (minimum description length) criterion[1]. In the method, the appropriate number of components 
is selected for each class on the basis of the likelihood for the training samples, penalizing too large number of components in order to avoid overfitting.

However, in pattern recognition, our main concern is classification. Such a class-independent selection may not be appropriate, especially in the case of the small number of training samples. For this problem, we have proposed a class-combinatorial selection method 2 . In the method, the combination of mixture models is evaluated on the basis of their class separability for the training samples, penalizing too complex classifier in order to avoid overlearning.

In our previous studies [2]3, the classification oriented criterion seemed to worked well. The proposed method marked the best recognition rate in some experiments. However, because of the low ability of the employed greedy algorithm, we could not show the relation between the optimal number of components in the criterion and the resultant recognition rate.

Therefore, in this study, we employ a genetic algorithm 4 in order to search the quasi-optimal solution. Experimental comparison of these three methods, the likelihood method, the class separability method with a greedy algorithm and the class separability method with a genetic algorithm, is carried out.

\section{Mixture Model Classifiers}

The mixture model classifier is one practical and effective implementation of Bayesian classifier. In this classifier, each class-conditional probability density function is approximated by the mixture of some component distributions. For this purpose, Gaussian distributions are generally used. Each probability density function is described as follows:

$$
p(\boldsymbol{x})=\sum_{k=1}^{K} c_{k} \mathrm{~N}\left(\boldsymbol{m}_{k}, \Sigma_{k}\right)(\boldsymbol{x})
$$

where $\boldsymbol{x}$ is a feature vector, $K$ is the number of components, $\mathrm{N}\left(\boldsymbol{m}_{k}, \Sigma_{k}\right)(\cdot)$ is a Gaussian distribution with a mean vector $\boldsymbol{m}_{k}$ and a covariance matrix $\Sigma_{k}$, and $c_{k}$ is the weight of the $k$ th component $\left(\sum_{k=1}^{K} c_{k}=1\right)$.

In this study, we estimate the mixture models using an iterative procedure called EM algorithm[ [5]. It enables local maximization of the following loglikelihood indirectly:

$$
L=\sum_{i=1}^{N} \ln p\left(\boldsymbol{x}_{i}\right)=\sum_{i=1}^{N} \ln \left\{\sum_{k=1}^{K} c_{k} \mathrm{~N}\left(\boldsymbol{m}_{k}, \Sigma_{k}\right)\left(\boldsymbol{x}_{i}\right)\right\},
$$

where $N$ is the number of training samples $\left\{\boldsymbol{x}_{i}\right\}$.

EM algorithm requires both the number of components and the initial components. For the initial components, we use fuzzy $c$-means method [6] with the fuzziness factor 1.6. Thus, once the number of components is given, we can obtain a mixture model by EM algorithm through the fuzzy clustering.

We estimate a mixture model for each class and combine them into a classifier through Bayesian theory. Therefore, in order to make best use of the mixture 
model classifier, we have to select the appropriate number of components for each class in advance.

\section{Selection of the Appropriate Number of Components}

In this study, the appropriate number of components is selected basically by repeating the estimation and evaluation of a mixture model, varying the number of components. The evaluation is made by MDL criterion[1]. There are two methods according to the calculation of the criterion.

\subsection{Selection Method Based on Likelihood}

In this method, we select the appropriate number of components by minimizing the following conventional formula:

$$
\mathrm{MDL}_{\mathrm{LH}}=-L+\frac{1}{2} m \log N,
$$

where $L$ is the log-likelihood of Eq.(1), and $m=(K-1)+K\{D+D(D+1) / 2\}$ is the number of parameters for specifying one mixture model. Here, $D$ is the dimensionality of the feature space.

In Eq.(2), the first term is low when the mixture model fits well to the training samples, and the second term is low when the mixture model is simple, i.e., the number of components is small. In another words, this method maximizes the likelihood, penalizing too large number of components.

We can obtain the mixture model that minimizes Eq. (2) simply by varying the number of components within a certain range. Note that this method is carried out for each class individually.

\subsection{Selection Method Based on Class Separability}

In order to take the class separability into account, we use another calculation of MDL criterion proposed by Kudo et al.[7]. In this method, both the classification ability for the training samples $S$ and the complexity of the classifier $c$ are evaluated in bit length. We select the classifier which minimizes the following formula:

$$
\mathrm{MDL}_{\mathrm{CS}}=L(S \mid c)+L(c)
$$

where $L(S \mid c)$ is the description length for encoding the labels of the training samples among $S$ misclassified by $c$, and $L(c)$ is the description length of $c$ itself.

The detail calculation of the above two terms for the mixture model classifiers is as follows:

$$
\begin{aligned}
L(S \mid c)= & N^{-} \mathrm{H}\left(N_{1}^{-}, \ldots, N_{M}^{-} \mid N^{-}\right)+\frac{1}{2}(M-1) \log _{2} N^{-} \\
& +N \mathrm{H}\left(N^{-}, N^{+} \mid N\right)+\frac{1}{2} \log _{2} N, \\
L(c)= & \alpha K D^{\beta} \log _{2} N,
\end{aligned}
$$


where $M$ is the number of classes, $N^{+}$and $N^{-}$are the number of samples classified correctly and incorrectly, respectively, and $\mathrm{H}\left(N_{1}, \ldots, N_{M} \mid N\right)$ is the entropy calculated by $-\sum_{i=1}^{M} \frac{N_{i}}{N} \log _{2} \frac{N_{i}}{N}$. Here, $K$ is the total number of components for all classes, and $\alpha$ and $\beta$ are constants for suppressing the superabundant effect of the second term. We experimentally determined $\alpha$ to be 0.235 and $\beta$ to be 0.876 for the mixture model classifiers. See the reference [7] for the detail.

In Eq.(3), the first term is low when the classifier can classify the training samples well, and the second term is low when the classifier is simple. In another words, this method maximizes the class separability, penalizing too complex classifier.

In this method, the search of the classifier which minimizes Eq. 3 is a classcombinatorial problem. We implemented the following two different algorithms for the mixture model classifiers.

\section{- Greedy Algorithm}

Start with one component for each class, and increase the number of components for a certain class so as to decrease Eq.(3). (proposed in the reference[2])

\section{- Genetic Algorithm 4}

Let $K_{1} K_{2} \cdots K_{M}$ be "chromosome", where "gene" $K_{i}$ is the number of components for the $i$ th class. Let the set of $C$ chromosomes be "population." Follow these steps.

1. Initialize the population.

Each chromosome is initialized with random numbers.

2. Select the better chromosomes.

a) For each chromosome, calculate $\mathrm{MDL}_{\mathrm{CS}}$ according to Eq.(3).

b) For each chromosome, let $\left(\mathrm{MDL}_{\mathrm{Worst}}-\mathrm{MDL}_{\mathrm{CS}}\right)$ be the "fitness" $f$. Here, $\mathrm{MDL}_{\mathrm{WORST}}$ is the worst $\mathrm{MDL}_{\mathrm{CS}}$ among the past $W$ generation.

c) Select $C$ chromosomes as a new population, according to the proportions of the fitness, i.e., the $j$ th chromosome is selected with the probability of $f_{j} / \sum_{k} f_{k}$. Note that the better chromosomes may be selected two or more times.

3. Generate the crossovers of the chromosomes.

a) For each chromosome, select a pair for the chromosome randomly, and generate uniform crossovers between them with the probability of $P_{c}$. This means the corresponding genes between the two chromosomes are exchanged randomly. By this process, $2 C$ chromosomes are generated.

b) Select $C$ chromosomes randomly from the $2 C$ chromosomes.

4. Mutate the chromosomes.

For each chromosome and each gene in the chromosome, modify the gene randomly with the probability of $P_{m}$.

5. Repeat $G$ times from step 2 to step 4 . 
In this study, we used 50 chromosomes for $C, 5$ generations for $W, 60 \%$ for $P_{c}, 1 / M$ for $P_{m}$ and 100 generations for $G$. In addition, we employed "elitist strategy," in which the best chromosome is necessarily saved to the next generation. This technique warrants $\mathrm{MDL}_{\mathrm{CS}}$ to be nonincrease. We adopt the final elite as the quasi-optimal solution of this problem.

\section{Experiments}

\subsection{Artificial Datasets}

First, we carried out experiments using two artificial datasets called "Cross" and "Ring." Each dataset is a 2-class problem in a 2-dimensional feature space. In Cross dataset, one class forms a cross and the other class comprises four squares surrounding the cross. These two classes are perfectly separable. In Ring dataset, one class forms the uniform ring with the inner radius of 2.147 and the outer radius of 3.030, and the other class is the Gaussian distribution with the mean vector at the origin and the unit covariance matrix. The radii were adjusted so as to the Bayesian recognition rate be $95.5 \%$. The example results obtained by the class separability method with the genetic algorithm are shown in Fig. 1 In these examples, 100 training samples were used per class.

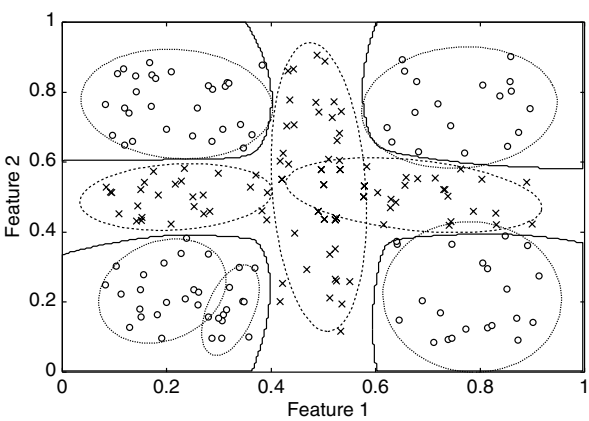

(a) Cross dataset $\left(\mathrm{K}_{1}=5, \mathrm{~K}_{2}=3\right)$

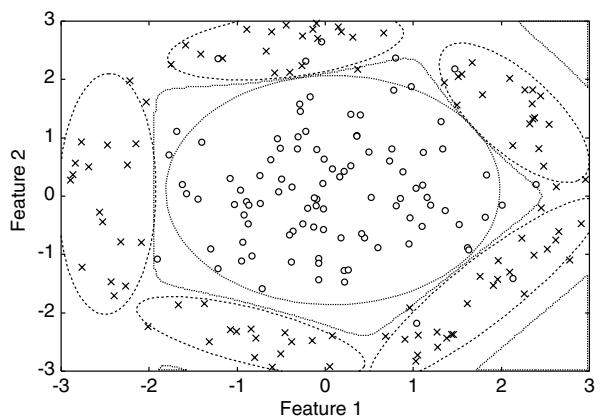

(b) Ring dataset $\left(\mathrm{K}_{1}=1, \mathrm{~K}_{2}=5\right)$

Fig. 1. Example results for (a) Cross dataset and (b) Ring dataset. The small symbols, the ellipses and the solid lines show the training samples, the component distributions and the classification boundaries, respectively.

For these two different types of datasets, we compared the three selection methods, using 20, 30, 40, 50, 100, 200 and 500 training samples per class. For each number of training samples, we carried out ten trials with different random seeds and let the averages be the results. The experimental results are shown in Fig. 2 and Fig. 3 .

Almost the same tendency was obtained in both datasets. In the case of the small number of training samples, the recognition rates of CS-Greedy and CS- 


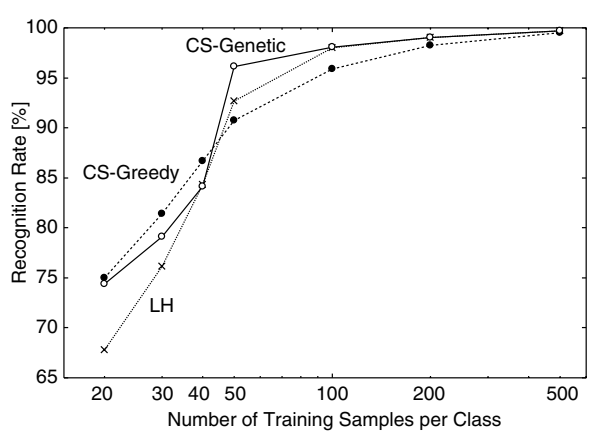

(a) Recognition Rate

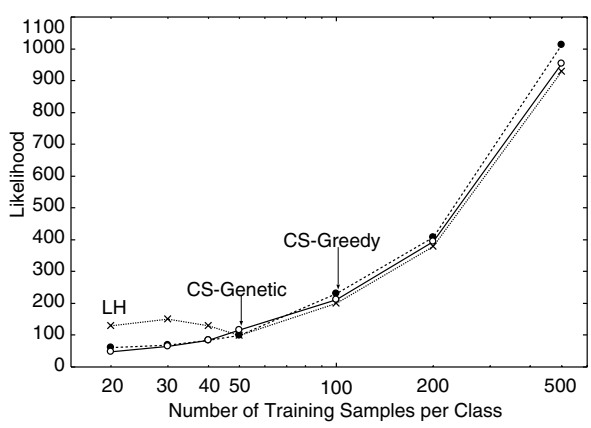

(c) Likelihood

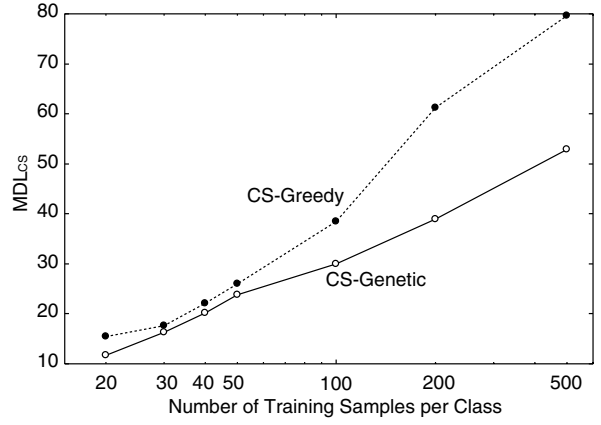

(b) MDLcs

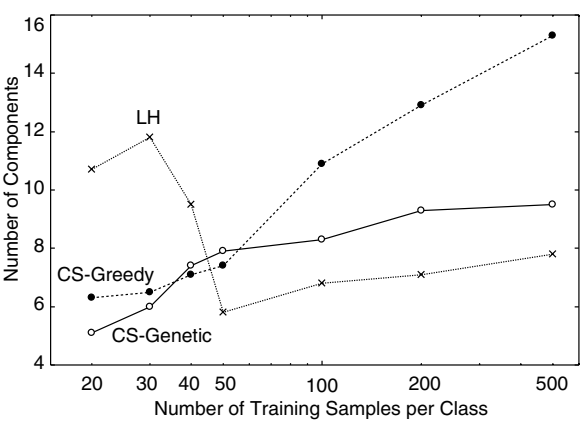

(d) Number of Components

Fig. 2. Experimental result for Cross dataset. CS-Genetic, CS-Greedy and LH correspond to the class separability method with the genetic algorithm, the class separability method with the greedy algorithm and the likelihood method, respectively. The likelihood in (c) shows the total sum of the log-likelihood for all classes, and the number of components in (d) shows the total sum of the number of components for all classes.

Genetic are higher than that of LH. However, in the case of the large number of training samples, LH is better than CS-Greedy. CS-Genetic almost agreed with LH. As for the minimization of $\mathrm{MDL}_{\mathrm{CS}}$, CS-Genetic marked lower $\mathrm{MDL}_{\mathrm{CS}}$ than CS-Greedy. This shows that CS-Genetic worked well.

Note that a high recognition rate was not always obtained from high likelihood models, especially in the case of the small number of training samples. However, in the case of the large number of training samples, high likelihood models marked a better recognition rate than the other models. This confirms LH can estimate the underlying distributions precisely in the case of the large number of training samples.

As for the number of components, LH selected many components, especially in the case of the small number of training samples. On the other hand, the both CS methods selected only a few components in that case. As the number of training samples increases, the number of components of $\mathrm{LH}$ converged within a certain range, that of CS-Greedy did not converge, and that of CS-Genetic con- 


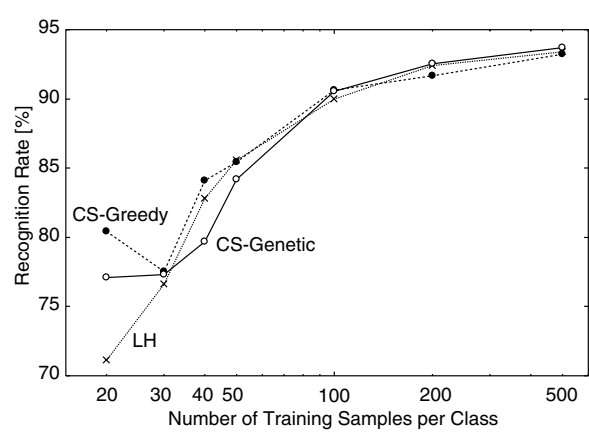

(a) Recognition Rate

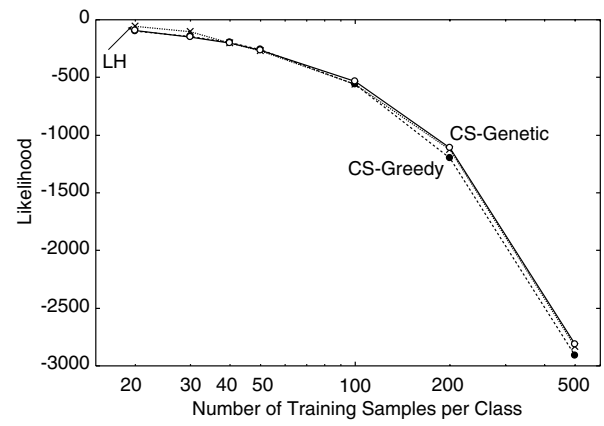

(c) Likelihood

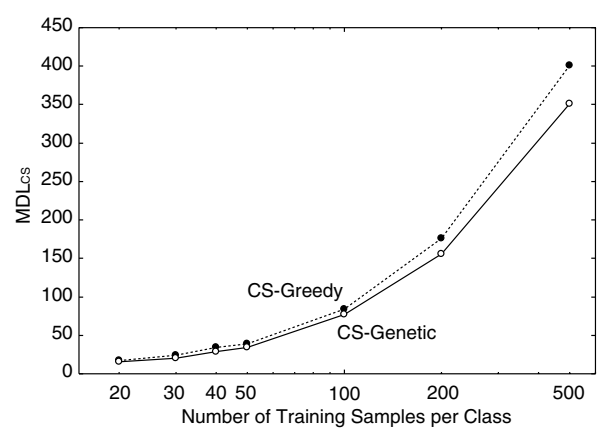

(b) MDLcs

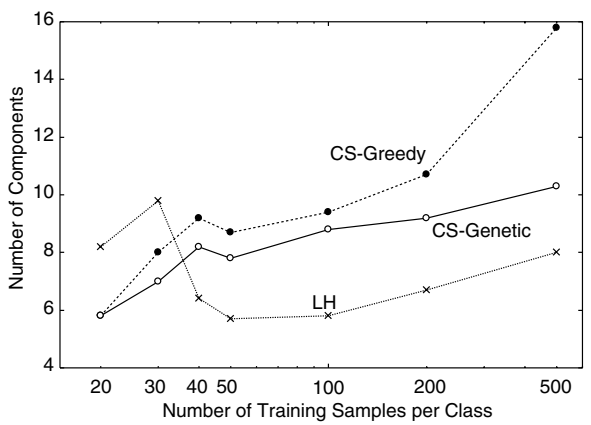

(d) Number of Components

Fig. 3. Experimental result for Ring dataset. CS-Genetic, CS-Greedy and LH correspond to the class separability method with the genetic algorithm, the class separability method with the greedy algorithm and the likelihood method, respectively. The likelihood in (c) shows the total sum of the log-likelihood for all classes, and the number of components in (d) shows the total sum of the number of components for all classes.

verged. From the consistency property of MDL criterion[1, the selected model have to be converged according to the increase of the number of training samples. The results also shows the CS-Genetic worked well from this viewpoint.

\subsection{Real Datasets}

We also compared the three methods using two real datasets called "Ship" and "Wine." Ship aims to distinguish 8 types of military ships with 11 features. See the reference 8] for the detail. Wine is taken from the UCI machine learning database 9 . Three wines are recognized by 13 chemical features. The total numbers of samples are 2545 and 178, respectively, and we estimated the recognition rates by 2 -fold and 10 -fold cross validations, respectively. Bayesian quadratic classifier and $k$-nearest neighbor method $(k=5)$ were also tested. The experimental results are shown in Table 1 and Table 2. In addition, the transitions of $\mathrm{MDL}_{\mathrm{CS}}$ and the recognition rate are shown in Fig. 4 and Fig. 5 . 
Table 1. Experimental results for Ship dataset. Here, $R$, \# and $L^{*}$ are the recognition rate, the total sum of the number of components for all classes and the total sum of log-likelihood for all classes, respectively.

\begin{tabular}{c|lccc} 
Classifier & $R[\%]$ & $\#$ & $L^{*}$ & MDL $_{\mathrm{CS}}$ \\
\hline Mixture(CS-Genetic) & 94.32 & 17.5 & 8771.52 & 476.02 \\
Mixture(CS-Greedy) & 94.32 & 17.5 & 8771.52 & 476.02 \\
Mixture(LH) & 94.07 & 34.0 & 14794.61 & - \\
Quadratic & 93.15 & - & - & - \\
$k \mathrm{NN}(k=5)$ & 86.71 & - & - & -
\end{tabular}

Table 2. Experimental results for Wine dataset. Here, $R$, \# and $L^{*}$ are the recognition rate, the total sum number of components for all classes and the total sum of loglikelihood for all classes, respectively.

\begin{tabular}{c|cccc} 
Classifier & $R[\%]$ & $\#$ & $L^{*}$ & MDL $_{\mathrm{CS}}$ \\
\hline Mixture(CS-Genetic) & 97.87 & 3.0 & -1646.71 & 60.01 \\
Mixture(CS-Greedy) & 97.87 & 3.0 & -1646.71 & 60.01 \\
Mixture(LH) & 30.11 & 23.2 & 4321.24 & - \\
Quadratic & 97.87 & - & - & - \\
$k N N(k=5)$ & 95.77 & - & - & -
\end{tabular}

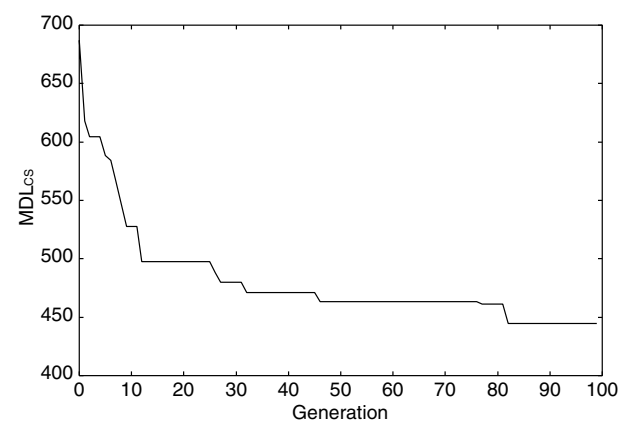

(a) MDLcs

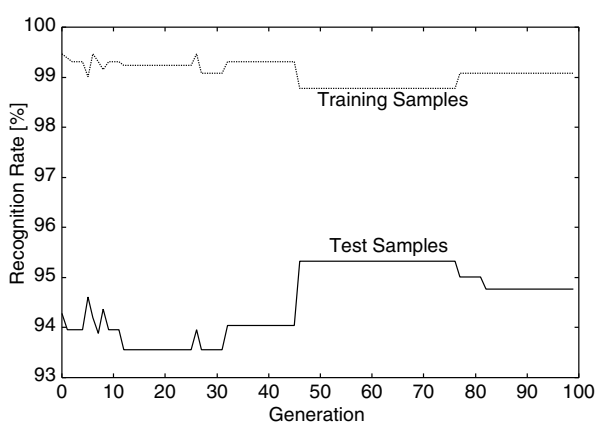

(b) Recognition Rate

Fig. 4. Transition of $\mathrm{MDL}_{\mathrm{CS}}$ and the recognition rate for Ship dataset (the first trial in the 2 -fold cross-validation).

In these datasets, we could not obtained a significant difference between CSGenetic and CS-Greedy. However, the both CS methods marked higher recognition rates than LH. As for the number of components, LH selected the larger number of components than the others. As a result, the likelihood of LH was also higher. From the result for the artificial datasets, this means the number of the training samples for these real datasets might be relatively small to their own difficulties. In addition, the recognition rates of both CS methods were higher 

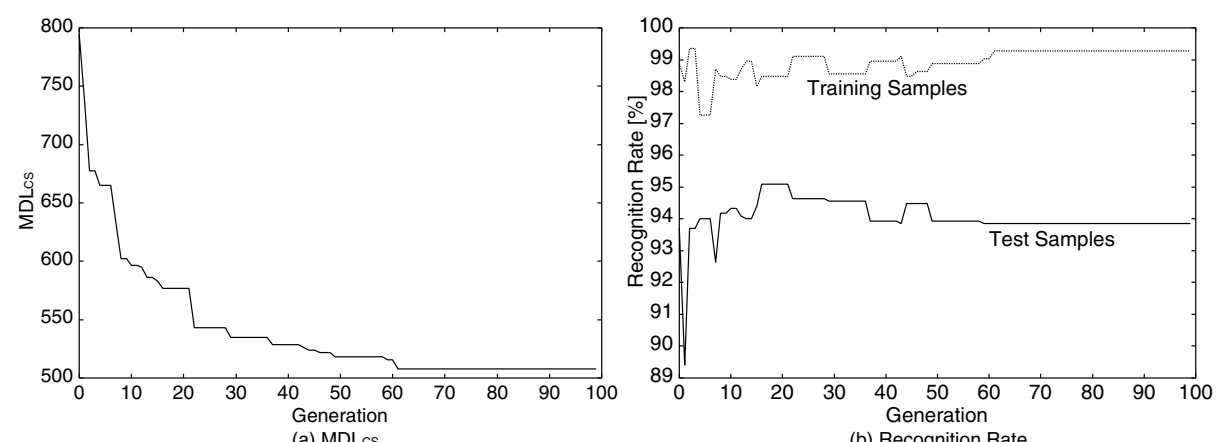

Fig. 5. Transition of $\mathrm{MDL}_{\mathrm{CS}}$ and the recognition rate for Ship dataset (the second trial in the 2 -fold cross-validation).

than those of the two conventional classifiers. This shows the superiority of the mixture model classifiers with the appropriate number of components.

From Fig. 4(a) and Fig. [5)(a), we can confirm that CS-Genetic worked well for minimizing $\mathrm{MDL}_{\mathrm{CS}}$. However, in Fig. 4(b) and Fig. 5(b), the peaks of the recognition rate curves for the test samples were appeared in the earlier generations. If the true model, i.e., the optimal mixture model classifier, is more complex than the selected model, this result agree with the consistency property of MDL criterion.

\section{Conclusion}

The genetic algorithm was employed in order to select the appropriate number of components for the mixture model classifiers. In this study, the appropriate number of components was selected on the basis of class separability, while the conventional method was based on likelihood. The combination of mixture models was evaluated by the classification oriented MDL criterion, and its optimization was carried out using the genetic algorithm. The effectiveness of this method was shown through the experimental results on some artificial and real datasets.

We have to carry out more experiments on the other datasets, in order to justify the classification oriented MDL criterion. Artificial datasets with varying the dimensionality of the feature space will be utilized for this purpose. The effect of the parameters in the genetic algorithm also should be investigated.

\section{Acknowledgement}

The authors are grateful to Professor Dr. Sklansky for providing the Ship dataset. 


\section{References}

1. Rissanen, J.: A Universal Prior for Integers and Estimation by Minimum Description Length. Annals of Statistics. 11 (1983) 416-431

2. Tenmoto, H., Kudo, M., Shimbo, M.: MDL-Based Selection of the Number of Components in Mixture Models for Pattern Recognition. Lecture Notes in Computer Science 1451, Advances in Pattern Recognition. Springer (1998) 831-836

3. Tenmoto, H., Kudo, M., Shimbo, M.: Determination of the Number of Components Based on Class Separability in Mixture-Based Classifiers. Proceedings of the Third International Conference on Knowledge-Based Intelligent Information Engineering Systems. (1999) 439-442

4. Holland, J. H.: Adaptation in Natural and Artificial Systems. University of Michigan Press (1975)

5. Dempster, A. P., Laird, N. M., Rubin, D. B.: Maximum Likelihood from Incomplete Data via the EM Algorithm. Journal of the Royal Statistical Society, Series B. 39 (1977) 1-38

6. Bezdek, J. C.: Cluster Validity with Fuzzy Sets. Journal of Cybernetics. 3 3(1974) $58-73$

7. Kudo, M., Shimbo, M.: Selection of Classifiers Based on the MDL Principle Using the VC Dimension. Proceedings of the 11th International Conference on Pattern Recognition. (1996) 886-890.

8. Park, Y., Sklansky, J.: Automated Design of Multiple-Class Piecewise Linear Classifiers. Journal of Classification. 6 (1989) 195-222

9. Murphy, P. M., Aha, D. W.: UCI Repository of Machine Learning Databases [Machine-Readable Data Repository]. University of California Irvine, Department of Information and Computation Science. (1996) 